\title{
3 Judenfeindschaft und Sprache: Verbale Macht- und Gewaltausübung
}

\subsection{Sprache als geistiges System und kommunikatives Handlungsinstrument}

Die Möglichkeit, mittels Sprache zu kommunizieren, gehört zu den wichtigsten geistigen und sozialen Fähigkeiten des Menschen. Sprachliche Kommunikation ist Grundlage jeder Gesellschaft. Alle wesentlichen sozialen Interaktionen, Strukturen und Institutionen basieren auf sprachlichen Prozessen. Sprachliche Äußerungen vermitteln allgemeine und individuelle Kenntnisse, speichern kollektives Wissen, drücken politische und ideologische Einstellungen aus, ermöglichen komplexe Denkprozesse und die Tradierung enzyklopädischer Informationen, aktivieren Gefühle, beeinflussen Bewusstseinsinhalte, erweitern Wissensrepräsentationen, lösen Assoziations- und Lernprozesse aus. Über das kognitiv und kulturell geprägte Kenntnissystem Sprache vermitteln Menschen ihre Wahrnehmungen, Eindrücke und Urteile, ihre Ideen, Vorstellungen und Überzeugungen, ihre Wünsche, Erwartungen und Ziele. Die Sprache ist das bei allen individuellen Unterschieden und subjektiven Ausrichtungen menschlicher Existenzen in einer Gemeinschaft von allen geteilte und benutzte, überindividuell verstandene Kenntnis- und Kodierungssystem. ${ }^{1}$ Ein System, das es ermöglicht, mittels regelgeleitet erzeugter Symbolstrukturen Informationen über die Welt nicht nur langfristig in einem Medium zu speichern, sondern diese auch an unsere Mitmenschen weiterzugeben. Ein System, das den Austausch von Informationen über Zeichen ermöglicht und damit gewährleistet, dass Menschen als Subjekte intersubjektiv $\mathrm{zu}$ anderen Kontakt aufnehmen und sich elaboriert mitteilen können. Mentale, d. h. nicht beobachtbare Gedanken werden mittels sprachlicher Formen in ein konkretes Medium gebracht und erhalten damit eine objektivierbare Dimension. Gemeintes wird zum Gesagten und damit Teil des gesellschaftlichen Prozesses und der sozialen Interaktion.

Mit Sprache beziehen wir uns auf die außersprachliche Welt. Wir beziehen uns auf Personen, Dinge, Sachverhalte, bilden Realitätsstrukturen ab, stellen verbal Zusammenhänge (kausaler, temporaler und räumlicher Art) dar, geben Urteile über die Wahrheit oder Falschheit von Aussagen. Sprache bildet Realität

1 Sprache umfasst als Kenntnissystem bestimmte Subsysteme (Lexik, Morphophonologie, Syntax, Semantik) mit spezifischen Regeln. Maßgeblich ist, dass immer Formen an Inhalte gekoppelt werden. Nur Formen allein sind bedeutungslos, die geistigen Inhalte dagegen lassen sich ohne Formanknüpfung nicht kommunizieren. 
aber nie einfach nur ab, da die Wahl der Mittel und die Art der Informationsanordnung stets schon eine spezifische Perspektivierung und Evaluierung vermitteln. Jede verbale Realitätsdarstellung kann so durch die Gestaltung der Äußerung variiert und der Sprecherintention gemäß geformt werden. Lexikon und Grammatik liefern ein Reservoir an unterschiedlichen Wörtern für Benennungen sowie die Möglichkeit, syntaktische Strukturen flexibel auszuwählen, um Inhalte semantisch und strukturell verschiedenartig darzustellen. Je nach Perspektive und Einstellung können z.B. Juden referenziell neutral, meliorativ, pejorativ, vage oder falsch bezeichnet werden als jüdische Mitbürger, Juden, Drecksjuden, Saujuden, Judenschweine, jüdische Parasiten, bestimmte Kreise, jene Religionsgemeinschaft, Ostküstenlobby, Zionisten, Israelis, Semiten etc. Durch die Synonymverwendung der Wörter Jude(n) und Israeli(s), die zwei Bedeutungen haben und sich außersprachlich auf verschiedene Referentengruppen beziehen, wird eine Identitätsrelation ausgedrückt, die de facto ${ }^{2}$ nicht richtig ist.

So erzeugt die jeweilige sprachliche Konstruktion immer eine subjektiv geprägte Darstellung von Realität. Sprachliche Äußerungen schaffen zum Teil eigene Realitäten, und über die Semantik, also die Bedeutungen der involvierten Strukturen werden ganze mentale Modelle ${ }^{3}$ erzeugt, die mit der außersprachlichen Realität nichts gemeinsam haben. Wie in dem (als klassisch für judeophobe Fiktionen zu bezeichnenden) Satz, der von Heinrich von Treitschke 1879 artikuliert und von den Nationalsozialisten 50 Jahre später in jeder Stürmer-Ausgabe als Schlagzeile reproduziert wurde:

„die Juden sind unser Unglück!““ (Treitschke 1879: 575)

Hiermit wird kollektiv allen Juden die Verantwortung für die Probleme und die Zukunft des deutschen Volkes zugesprochen (ein Urteil, das angesichts der damaligen Realität ebenso falsch wie grotesk ${ }^{4}$ war; s. hierzu Kap. 4.2). Zudem wird durch die additive Gegenüberstellung grammatisch die Abgrenzung von Juden und Deutschen verbalisiert. Die aktuelle Äußerung (2) aus dem Jahr 2007:

2 Nicht nur, dass selbstverständlich nicht alle Juden Israelis sind, sondern Deutsche, Franzosen, Amerikaner etc., es sind auch nicht alle Israelis Juden: Es gibt arabische (muslimische und christliche) Israelis, die Staatsbürger von Israel sind.

3 Äußerungen evozieren nicht nur einzelne Konzepte, sondern komplexe Schemata, d. h. Konzeptverbindungen, die als Modellkonstruktionen mentale Realitätsstrukturen widerspiegeln (s. Schwarz ${ }^{3} 2008$ : $115 \mathrm{ff}$.).

4 Im 19. Jahrhundert stellten die Juden ca. ein Prozent der deutschen Bevölkerung und sahen sich zudem massiven Anfeindungen und sozialen Behinderungen ausgesetzt. Von einer wesentlichen Einflussnahme, die dem deutschen Volk hätte Schaden zufügen können, konnte 
„Juden sind das Übel der Menschheit und bedrohen den Weltfrieden.“ [ZJD_29.05.2007_Sch_002] $]^{5}$

spiegelt ebenfalls die realitätsverzerrende und zugleich realitätskonstituierende Komponente wider, die durch den hyperbolischen und generischen Sprachgebrauch entsteht. Ein Feind- und Gefahren-Szenario wird etabliert, das allein im Kopf des Sprachproduzenten existiert, jedoch über die Verbalisierung als weltabbildend kommuniziert wird.

Sprachliche Äußerungen erzeugen also beobachterabhängige Text-Welten, kognitive Zwischenebenen, die oft kein Äquivalent in der realen Welt ${ }^{6}$ haben. Die realitäts- und gegenstandskonstituierende Funktion von Sprache wird besonders deutlich, wenn wir auf fiktive Texte blicken. Der Produzent eines literarischen Werkes kreiert mittels sprachlicher Strukturen eine eigenständige, komplexe, in sich durchaus kohärente, aber fiktive Wirklichkeit. Ähnlich ist es bei den Konstrukten der Judenfeindschaft: Die zum Teil seit Jahrhunderten tradierten judeophoben Stereotype haben mehrheitlich keinerlei reale Basis, sondern sind kognitiv hergestellte und sprachlich vermittelte Fiktionen (s. hierzu Kap.4), die als Konzeptualisierungsmuster in den Köpfen ihrer Benutzer gespeichert sind (oder kulturell als kommunikativer Kode in den Sprachformen konserviert werden). Die Weltverschwörungspläne in den Protokollen der Weisen von Zion oder die seit dem Mittelalter kodierten Vorstellungen von Juden als kindermordenden Blutkultanhängern sind nachweislich reine Phantasieprodukte, aber sie werden weltweit

also nicht einmal im Entferntesten ausgegangen werden (s. Friesel 1990). Genauso realitätsfern war die Aussage in der NS-Zeit.

5 Dieses Kürzel zeigt an, dass es sich bei einem Beispiel um einen authentischen Text aus dem E-Mail-und-Brief-Korpus handelt. In diesem Fall steht ZJD für Zentralrat der Juden in Deutschland, mit dem Datum, an dem der ZJD die E-Mail erhielt; für den Namen des Schreibers bzw. der Schreiberin wurde ein anonymisierendes Kürzel eingesetzt. Mit IBD beginnende Kürzel hingegen markieren Zuschriften, die bei der Israelischen Botschaft in Deutschland eingegangen sind.

6 Auch wenn hier nicht ein naiver Realismus angenommen wird, sondern die Annahme des Konstruktivismus, dass Realität immer im Kopf des Betrachters entsteht, so können wir doch nicht darauf verzichten, Realitätseinschätzungen in Form von Vergleichen vorzunehmen, da wir sonst in unserer Gesellschaft in eine totale Unverbindlichkeit abgleiten würden. Wir nehmen in unserem Leben immer an, dass es eine Realität gibt, die als Basis für Wahr-oder-falsch-Urteile dient. Die reale Welt hat für uns den Charakter eines verbindlichen, normalerweise nicht in Frage gestellten Bezugssystems; sie liefert die Grundlage für unsere Bewertungen, unsere Wahrheitsansprüche, unsere rechtlichen Entscheidungen. Wir orientieren uns an dieser Realität und es gehört zu unseren kognitiven Fähigkeiten, dass wir normalerweise die reale Welt und die fiktionalen Welten auseinanderhalten können. 
von Millionen von Menschen geglaubt und über diverse Texte ${ }^{7}$ in der kommunikativen Praxis erhalten und tradiert. Verdichten sich die judeophoben Konzepte zu einem komplexen Glaubenssystem, entsteht ein für Fakten geschlossenes Weltbild, in dem sich pseudo-rational alles kohärent zusammenfügt. Innerhalb des Systems wird jede Information plausibel integriert bzw. als passend umgedeutet: Werden z. B. Juden Opfer von verbaler und/oder nonverbaler Gewalt, so ist dies aus der systemimmanenten Sicht des antisemitischen Betrachters immer deren eigene Schuld.

Aggressive Sprache und Judenfeindschaft verbindet eine lange Symbiose. Die Sprachgebrauchsgeschichte zeigt, wie Juden über die Jahrhunderte hinweg (trotz wechselnder sozialer Konstellationen) weitgehend semantisch homogen abgewertet und ausgegrenzt wurden. Die Sprache erweist sich als kulturelles Erbe und kollektives Wissensreservoir, als Konservierungsmittel für Mythen und Stereotype, die unhinterfragt tradiert werden, die zum kollektiven Wissen einer Gesellschaft gehören, obgleich ihr Geltungsanspruch nicht empirisch unterlegt ist. Sprache trägt, lenkt und prägt maßgeblich unser Denken. Sprache ermöglicht, Identität auszudrücken (Ich als junger Deutscher der Nachkriegsgeneration), nationales Selbstbewusstsein zu erfahren (Ich bin stolz auf mein Land Deutschland), Sprache stiftet Gemeinsamkeit und Identität, grenzt gleichzeitig ab; individuelles und kollektives Ego und Alter Ego werden mittels Sprache über gruppenzuweisende Merkmale definiert (vgl. 'Wir Deutschen versus Ihr Juden'). Sprache vermittelt Urteile und Bewertungen (vgl. Israel ist ein Unrechts- und Apartheidstaat), steuert Meinungsbildungsprozesse (vgl. Wir haben ein Kritiktabu), transportiert bzw. reaktiviert Stereotype (vgl. Alle Juden sind geldgierig), benennt, weckt bzw. beeinflusst Gefühle (vgl. Aus großer Sorge um den bedrohten Weltfrieden muss ich schreiben), konstruiert extrem negative Vorstellungen (Pestbeule Judentum), Bedrohungen (das internationale Judentum will uns vernichten) und fiktive Analogien (Mit SS-Methoden geht das israelische Militär vor). Sprache kann Feindbilder ${ }^{8}$ erzeugen, aus einem Individuum oder einer Gruppe eine schädliche, böse Figur oder Instanz machen (wie in „Die Juden zerstören Deutschland“, E-Mail an den ZJD 2009). Texte erschaffen oft nicht nur ihre eigenen Realitätsstrukturen („Alle Israelis sind Mörder“, E-Mail an die IBD 2008; „Juden beherrschen die Presse“, an

7 Die Protokolle der Weisen von Zion sind in einigen arabischen Ländern ebenso oft gedruckt und verbreitet wie Hitlers Mein Kampf. Auf rechtsradikalen Homepages werden die Protokolle als Beweis für die antisemitische Ideologie zitiert. In der Charta der Hamas findet sich unter Artikel 32 der intertextuelle Verweis auf die Richtigkeit der Protokolle der Weisen von Zion. S. hierzu ausführlicher Wistrich (2011).

8 Vgl. Marcuse (1984: 303): „Sprache definiert und verdammt den Feind nicht nur, sie erzeugt inn auch; und dieses Erzeugnis stellt nicht den Feind dar, wie er wirklich ist, sondern vielmehr, wie er sein muß, um seine Funktion [...] zu erfüllen.“ 
den ZJD 2002), sondern liefern zugleich sehr spezifische Bewertungsmaßstäbe bzw. -systeme, in denen Kategorien wie Gut und Böse klar definiert sind (,Juden sind das Übel der Welt“).

Es reicht allerdings nicht, die referenzielle Darstellungsfunktion der Sprache und die ihr zugrundeliegenden Konzeptualisierungen zu analysieren, wenn man das gesamte Informations- und Wirkungspotenzial von sprachlichen Äußerungen erfassen will. Über die expliziten Referenzialisierungen als Verbalmanifestationen hinaus vermitteln Texte nämlich oft Informationen, die implizit enthalten sind, deren problemlose Rekonstruierbarkeit aber vorausgesetzt wird. Ein Satz wie (3) (der in diversen Varianten z. B. in rechtsgerichteten Publikationen wie der National-Zeitung zu lesen ist):

„Die Banker an der Ostküste beeinflussen Amerikas Politik.“

enthält keinen Ausdruck, der in irgendeiner Weise auf Juden Bezug nimmt. Die vage Nominalphrase „Banker an der Ostküste“ jedoch ist eine (mittlerweile schon als Floskel benutzte) Paraphrase für amerikanische Juden. Ähnlich funktioniert es mit referenziell unterspezifizierten Ausdrücken und Phrasen wie jene Lobby, die in unserem Land das Sagen hat oder die Religionsgemeinschaft, die uns am Wickel hat. Die Leser entsprechender Textstellen erschließen kontextuell sofort aufgrund ihres Weltwissens, wer gemeint ist.

Eine an den Zentralrat der Juden in Deutschland (in den letzten Jahren viel) gestellte Frage wie:

$$
\text { „Warum ziehen sie nicht nach Israel?“ }
$$

impliziert, dass der Sprachproduzent deutsche Juden nicht als Deutsche, sondern als Israelis sieht. Solche indirekten Sprechakte ${ }^{9}$ werden oft benutzt, um Tabus zu umgehen und sich vor Sanktionen zu schützen. Da nach 1945 ein

9 Indirekte Sprechakte drücken nicht expressis verbis aus, was tatsächlich gemeint ist. Es sind Formen der Umwegkommunikation: Der Sprecher sagt x, meint aber y. Die durch die grammatische Oberfläche vermittelte Funktion entspricht nicht der intendierten Funktion. So kann ein Inhalt als Frage in den Raum gestellt werden, tatsächlich aber ist der Sprecher von der Wahrheit der Aussage überzeugt und meint die Frage als Feststellung. Bei indirekten Sprechakten gibt es über die wortwörtliche Bedeutung der Äußerung hinaus noch einen zusätzlichen Sinn. Jeder kommunikativ kompetente Sprecher/Hörer einer Sprachgemeinschaft ist in der Lage, durch Weltwissensaktivierung und Kontextinformationen diesen Sinn zu verstehen. Es gehört zu den grundlegenden Prinzipien sprachlicher Informationsvermittlung, dass nicht nur das expressis verbis Gesagte, sondern auch das Erschließbare höchst bedeutungsvoll sein kann (s. u. a. Liedtke 1995, Wagner 2001). 
offener Antisemitismus und die Leugnung des Holocaust nicht nur verpönt sind, sondern als Volksverhetzung auch strafrechtlich geahndet werden, werden neben der bereits erwähnten referenziellen Unterspezifikation indirekte Formen der Informationsvermittlung benutzt, z. B. rhetorische Fragen (Sollen wir Deutschen ewig büßen? Wer braucht denn allen Ernstes ein Mahnmal?), Anspielungen und Wortspiele (USrael, IsraHölle, Schwindlers List). Insbesondere der Typ der referenziellen Verschiebung ist frequent: Referiert wird auf Israel, gemeint sind aber alle Juden. So muss nicht ein einziges Mal das Wort Jude oder jüdisch in einer Äußerung vorkommen, um judenfeindliche Inhalte zu vermitteln. Vielmehr vertrauen die Produzenten darauf, dass ihre Rezipienten den gemeinten Sinn über Schlussfolgerungen (als Implikaturen) erschließen können. Implikaturen sind nicht explizit formulierte, aber über den Inhalt der Äußerung im Kontext erschließbare Bedeutungen. Da es sich hierbei um rein mentale Schlussfolgerungen handelt, können sie vom Sprachproduzenten annulliert bzw. zurückgezogen werden, wenn eine brisante Situation entsteht. Der Produzent verweist dann auf das wörtlich Gesagte (und leugnet das kognitiv zu Rekonstruierende). So können nach der Artikulation eines Satzes wie Die Israelis sind eine verbrecherische, blutrünstige Mörderbande unterm Davidstern! die möglichen Implikaturen 'Israel hat keine Existenzberechtigung' oder 'Israelis sind Juden und Juden sind Mörder' bei Bedarf negiert werden. Die Verwendung indirekter Sprechakte ist also eine kommunikative Vorsichtsmaßnahme auf Seiten der Sprachbenutzer.

Mit Sprache wird Realität nicht nur spezifisch dargestellt, sondern mit Sprache wird direkt in die Realität eingegriffen. Sprache ist Handlungsinstrument ${ }^{10}$ und kann wie eine Waffe benutzt werden, um Menschen Schaden zuzufügen, sie zu kränken, zu beleidigen, zu verunglimpfen, sie auszugrenzen, ihnen zu drohen (s. hierzu ausführlich Kap.10.1). Mit Sprache kann man nicht nur zur Gewalt aufrufen, sondern ihr Gebrauch kann selbst eine Form von Gewaltausübung sein. Kommunikativ unterscheidet man verschiedene Typen von verbalen Handlungen (vgl. Searle 1969, Meibauer 2001, Bublitz 2009, Schwarz-Friesel 2007):

Mit Direktiva ist ein unmittelbarer Appell an den Adressaten verbunden: Eine Äußerung wie „Verlassen Sie Deutschland!“ (an den ZJD) fordert zu etwas auf; die außersprachliche Welt soll der Sprache folgen, nach ihr ausgerichtet werden. Mit Kommissiva wie Drohungen („Ich werde alles tun, um meine Mitmenschen gegen Juden aufzuhetzen“, E-Mail an die IBD 2007, oder „Wir schlagen euch alle tot!“, E-Mail an den ZJD 2005) wird ebenfalls versucht, Realität an die Sprache

10 Dass mit Sprache Handlungen vollzogen werden, gilt spätestens seit der Sprechakttheorie als unumstrittene Grundannahme jeder Sprach- und Kommunikationstheorie (vgl. hierzu die Klassiker Austin 1962 und Searle 1969; s. auch Habermas 1981). 
anzupassen, indem sie Zukünftiges prädizieren und damit u.a. die Adressaten ängstigen oder unter Druck setzen. Assertiva/Repräsentativa haben die Funktion, als Feststellungen Realität darzustellen; die Sprache richtet sich nach der Welt und bildet sie ab. Der Sprecher legt sich auf die Wahrheit oder Falschheit der Aussage(n) fest. Wenn repräsentative Sprechakte jedoch realitätsverzerrend oder verfälschend benutzt werden, fungieren sie als Beleidigungen, Diffamierungen und Diskriminierungen wie in Alle Juden sind geldgierig oder Israel ist ein NS-Staat. Eine assertive Äußerung wie Den Holocaust hat es nie gegeben verdreht die Realität, stellt aber darüber hinaus auch eine affektive Verletzung der Opfer(nachkommen) dar. Holocaustleugnungen sind nicht nur Geschichtsfälschungen, sondern sie beinhalten zugleich immer auch die Unterstellung, die Anderen seien Lügner. Zugleich verhöhnen sie die Erinnerung an das Leid der Opfer. Mit einer Feststellung können somit zugleich mehrere Handlungen gleichzeitig vollzogen werden. Das Handlungspotenzial der Sprache zeigt sich auch bei Expressiva, d.h. gefühlsbekundenden Sprachhandlungen, in denen der Produzent seine Einstellung zu einem bestimmten Sachverhalt artikuliert, wie in „Mir wird schlecht, wenn ich an Juden denke“ (Postkarte an die IBD 2006) oder „Ekelhaft, das Judenpack! “ (an den ZJD 2003). Auch diese Sprechakte üben Gewalt aus, da sie die Adressaten beleidigen und verletzen, ihre Würde angreifen.

\subsection{Macht der Sprache als Gewalt durch Sprache}

Wenngleich Macht ${ }^{11}$ und Gewalt nicht gleichzusetzen sind, so sind sie doch untrennbar miteinander verbunden. Gewalt ${ }^{12}$ ist ein bestimmter Subtyp der mög-

11 Das Thema nonverbale Gewalt ist in den letzten Jahren von Philosophen, Politik-, Sprach- und Sozialwissenschaftlern sowie Psychologen vor allem im Zusammenhang mit der sogenannten Hass-Rede erörtert worden (vgl. u. a. Butler 1998, Corbineau-Hoffmann/Nicklas 2000, Erzgräber/Hirsch 2001, Haubl/Caysa 2007, Krämer/Koch 2010). Die bisherige Forschung hat sich dabei jedoch primär auf theoretische, sprachphilosophische Reflexionen und die Erörterung juristischer Konsequenzen beschränkt (vgl. Zimmer 2001, Delgado/Stefancic 2004, Herrmann et al. 2007). „Gewalt in der Sprache“ wird als eine von der physischen Gewalt abzugrenzende mentale Komponente mit ihren möglichen Auswirkungen erörtert (s. Krämer/Koch 2010). Sowohl die emotionale Komponente, als das den Manifestationsformen zugrundeliegende Phänomen, als auch die verbale Komponente wurden nicht hinreichend analysiert (vgl. aber Kiener 1983, van Dijk 1984 und 2002, Jäger ${ }^{3}$ 2001, Reisigl/Wodak 2001, Graumann/Wintermantel 2007, Havryliv 2009, Meibauer 2012). Es mangelt bislang insbesondere an empirisch fundierten Untersuchungen, die dezidiert Aufschluss über aktuelle verbale Gewaltformen in ihren verschiedenen Facetten geben können (s. hierzu auch kritisch Haubl/Caysa 2007 und Schwarz-Friesel 2012b).

12 Der juristische Gewaltbegriff erfasst im Wesentlichen die körperliche Gewalt. Vgl. StGB 
lichen Machtaktivitäten, dem eine Täter-Opfer-Struktur inhärent ist. Sprache wird dabei zur Waffe. Verbale Gewalt ist eine destruktive Form der Machtausübung. Sie erfolgt bewusst und intentional mit dem Ziel, den/die Anderen zu kränken, zu beleidigen, auszugrenzen etc. Dies trifft auf die verbale, aber auch die nonverbale Gewalt ${ }^{13} \mathrm{zu}$. Sprache hat als Handlungsinstrument das Potenzial, Menschen kognitiv wie emotional zu verletzen und ihnen nicht nur individuell, sondern auch gesellschaftlich Schaden zuzufügen. Ihr Einsatz zielt einerseits auf die Verletzbarkeit von Menschen, andererseits auf Beeinflussung bzw. Gewinnung gleichgesinnter Rezipienten. Sprachliche Äußerungen aktivieren, reaktivieren und konstruieren Gedankengut, das die öffentliche Meinung und das kollektive Bewusstsein massiv und nachhaltig beeinflussen kann.

Mit sprachlichen Äußerungen werden Menschen als Individuen und/oder als Mitglieder von Gruppen angegriffen, beleidigt, verhöhnt, bedroht und diffamiert. In alltäglichen Kommunikationssituationen kommt es immer wieder zu Formen der aggressiven Gewaltausübung durch Sprache. Aggressivität (deren Basis eine negative, emotionale Einstellung ist, die selbstgerichtet oder fremdgerichtet sein kann) zeigt sich verbal in feindseligen Handlungen, deren Ziel die kognitive, emotionale oder soziale Schädigung einer Person ist. Spielt bei einer Verbalattacke der Bezug auf die Gruppe bzw. Gemeinschaft, der diese Person angehört, eine Rolle, handelt es sich um verbale Diskriminierung. Verbale Diskriminierung ist eine Form von Gewaltanwendung, die das Machtpotenzial von Sprache nutzt, um gesellschaftliche Gruppen (sei es aufgrund ihrer Ethnienzugehörigkeit, ihres Geschlechts, ihrer sexuellen Ausrichtung, ihrer Religion, ihres Alters oder ihrer Herkunft) von der (vom Aggressor als normal etablierten) Mehrheitsgesellschaft semantisch auszugrenzen und abzuwerten. Die gruppenbezogene Ausgrenzung basiert auf der fundamentalen sozialpsychologischen Unterscheidung von Eigenund Fremdgruppe, die sich auf allen Ebenen menschlicher Sozialisierung zeigt. Von nur aggressiven, aber nicht notwendigerweise diskriminierenden Sprechakten unterscheidet sich die Diskriminierung als verbale Handlung durch die Komponente 'gruppenbezogen'. Dieses semantische Merkmal ist wesentlich für die Bestimmung, ob eine Sprachhandlung Kritik, Beleidigung oder (die soziale oder ethnische Ungleichheit einer Gruppe betonende) Diskriminierung ist.

Dass mit sprachlichen Äußerungen aktiv und bewusst Handlungen vollzogen werden, ist nur eine Dimension ihres Macht- und Gewaltpotenzials. Sprache

$\S 240 \mathrm{ff}$. Nonverbale Formen der Gewalt werden u. a. unter StGB § 130 zur Volksverhetzung aufgeführt.

13 Dass nonverbale Gewalt im Sinne von Herrschaftsausübung Menschen als Opfer stigmatisiert, diskriminiert und zugleich Bewusstseinsmanipulation darstellen kann, zeigt sich besonders deutlich bei staatlich kontrollierter Sprachlenkung (vgl. z. B. die NS-Propaganda). 
hat auch Macht, weil sie ein Instrument der Beeinflussung und Lenkung unserer Gedanken und Gefühle ist, weil durch sie diese Manipulation ausgeübt werden kann, ohne dass sie bewusst wird. Die Bedeutung von Wörtern schleicht sich oft unbemerkt in unseren Geist ein, sie hinterlässt Spuren, löst Assoziationen aus, prägt zum Teil langfristig Einstellungen und Gefühle. Über die Semantik der Wörter und Sätze (wie „Die rachsüchtigen Juden nutzen die Deutschen schamlos aus!“", E-Mail an die IBD 2009) werden spezifische Repräsentationen und Wertungen aktiviert, die meinungsbildend sein können. Sprache übt somit kognitive Macht ${ }^{14}$ aus, die soziale Folgen haben kann. Die Macht des Wortes, von der Freud (1926: 13) sprach, ist heute empirisch nachgewiesen: Wörter aktivieren in unserem Langzeitgedächtnis in wenigen Millisekunden mentale Repräsentationen, setzen Gefühle frei, lassen spezifische mentale Bilder ${ }^{15}$ entstehen (s. u. a. Miller 1995, Schwarz-Friesel 2007 und 2008, Aitchison 42012). Menschen lassen sich mehr beeinflussen, als sie denken, da viele Prozesse unbewusst ablaufen und sich der Kontrolle entziehen. ${ }^{16}$ Bedeutungszuordnung ist als mentaler Prozess nicht zu unterdrücken: Menschliche Sprachrezeption zeichnet sich dadurch aus, dass sie automatisch, wie ein Reflex, abläuft. Wir können nichts dagegen tun (außer uns fest die Ohren bzw. Augen zuzuhalten). Unser Gehirn reagiert auf sprachliche Impulse in Millisekunden und ordnet den Ausdrucksformen blitzschnell Inhalte zu. Sprachliche Äußerungen aktivieren sofort mentale Repräsentationen in unserem Gedächtnis. Eine kontrollierte Einflussnahme ist nicht möglich (s. hierzu Fodor 1983, Schwarz ${ }^{32008: ~} 167$ ff.). Erst nach der automatischen Bedeutungszuordnung sind wir kognitiv in der Lage, uns mit den wahrgenommenen Inhalten kritisch und kontrolliert auseinanderzusetzen. Diese sind aber bereits im Arbeitsgedächtnis gespeichert und können unbewusst auf uns wirken. Sprachverarbeitung läuft auf vielen Ebenen ab, von denen einige für das Bewusstsein nicht direkt zugänglich sind. So ist es möglich, dass emotionsaktivierende Wörter oder Sätze Spuren im Langzeitgedächtnis hinterlassen oder

14 Macht im positiven wie im negativen Sinne: Die Macht des Wortes zeigt sich in einem Gedicht, das uns zu Tränen rührt, einem Roman, der uns aufwühlt, begeistert, einem Liebesbrief, der uns glücklich macht, einer Rede, die uns ängstigt, einem Text, der uns wütend macht.

15 Die Rezeption bestimmter Wörter kann nicht nur Emotionen verstärken, sondern sogar Schmerzen auslösen (vgl. Richter et al. 2010).

16 Ein Großteil der mentalen Prozesse in der menschlichen Kognition verläuft unbewusst und automatisch, unbeeinflusst von Intention und Verstand, unkontrollierbar von unserem bewusst einsetzbaren Willen (s. hierzu z. B. Schwarz ${ }^{3} 2008$ : 162 ff., Kahneman 2011). Die Semantik sprachlicher Äußerungen kann dementsprechend Ressentiments wecken oder verstärken, ohne dass dies bewusst wird oder verhindert werden kann. 
Assoziationsverbindungen etablieren können, ohne dass der Hörer/Leser dies bemerkt oder gar möchte.

Ein einzelnes Wort kann in den Köpfen von Menschen ein komplexes Szenario aktivieren: Apartheid bedeutet 'institutionalisierte, staatliche Rassenbzw. Ethnien-Trennung'. Hören oder lesen wir dieses Wort, aktivieren wir sofort das mentale und prototypische Bild des ehemaligen Südafrika mit getrennten Schulen, Restaurants, Parkbänken, mit diskriminierenden Gesetzen und staatlichen Repressalien. Die empirische Kognitionswissenschaft zeigt, dass die mentale Prozedur des Vergleichens zu den wesentlichen Prozessen der menschlichen Kognition gehört. Mittels des bewussten wie unbewussten Vergleichens versucht der menschliche Geist, z. B. mit neuen oder schwer zu begreifenden Erfahrungen oder Objekten umzugehen, indem Unbekanntes durch Analogie verständlicher wird. Wird also Israel als Apartheidregime bezeichnet, ist ein falscher Analogieschluss schnell gezogen, das Land grob verzerrt stigmatisiert. Wörter sind oft wie Pfeile, sie bohren sich in unser Bewusstsein ein. Juden als Parasiten, Rattenpack oder Untermenschen zu bezeichnen, bedeutet, sie als dehumane Wesen zu kategorisieren und als Menschen zu entwerten. Wörter können aber auch wie Gift wirken, sie tröpfeln Urteile ein, die langfristig Schaden anrichten können. Auschwitzkeule und Holocaustindustrie haben dieses Potenzial: Die Erinnerung an den Holocaust wird durch diese Komposita in den semantischen Kontext der Instrumentalisierung gestellt. Nicht der Zivilisationsbruch und das Leid der Opfer stehen im Fokus, sondern die ihnen unterstellte Nutznießung. Dadurch wird dem Verbrechen die entscheidende Relevanzdimension genommen bzw. diese wird in den Hintergrund gerückt. Häufig benutzte Sprachgebrauchskonstruktionen können zu Normalisierungseffekten führen: Wenn drastische Vokabeln (Verbrecherstaat, Mörderregime, Gewaltorgien), hyperbolische Phrasen (schlimmste Kriegsverbrecher, übelste Schandtaten) und NS-Vergleiche immer wieder in der sogenannten Israel-Kritik benutzt werden und unwidersprochen bleiben, wird deren Unverhältnismäßigkeit nicht mehr registriert, eine Habitualisierung setzt ein. Mit NS-Vergleichen geht zudem immer auch eine Relativierung des Holocaust und die Verhöhnung der Opfer einher (s. SchwarzFriesel 2007: 195 ff.). Der inflationäre Gebrauch des Wortes Holocaust stellt nicht nur die Singularität dieses Verbrechens in Frage, er kann auch zu Bedeutungsveränderungen führen: Von der Bedeutung 'Mord an den europäischen Juden' könnte es langfristig zur Bedeutungserweiterung im Sinne von 'Mord, Gewalttat, ethnische Säuberung' kommen. Dadurch würden sich langsam die Erinnerung an und das Bewusstsein für die unikalen Dimensionen dieses Zivilisationsbruchs auflösen. Wenn das Lexem Jude in verschiedenen Diskursen (in Internetforen, in der Jugendsprache auf den Schulhöfen etc.) nicht als Appellativum mit seiner referenzfestlegenden Bedeutung 'Mitglied der jüdischen Glaubensgemeinschaft', 
sondern als Schimpfwort benutzt wird, kann zum einen über die Kommunikation eine Bedeutungsverschiebung erfolgen und das Wort in bestimmten Kreisen zum Stigma $^{17}$ werden, zum anderen können auch im alltäglichen Sprachgebrauch ${ }^{18}$ negative Konnotationen entstehen. Häufig benutzte syntaktische Verbindungen des Adjektivs jüdisch mit Nomen, die sich auf Gewalt, Militäraktionen und Krieg beziehen, können solche Verbindungen usuell werden lassen und damit ein semantisches Netz anti-jüdischer Inhalte entstehen lassen bzw. alte Vorurteilsstrukturen reaktivieren. Das „Gerücht über die Juden“, wie Adorno ([1951] 1980: 123) das Phänomen des Antisemitismus nannte, wird durch solche Diskursmerkmale am Leben erhalten, es bekommt Verstärkung über Spekulationen, Mutmaßungen, gezielte Verleumdungen, aber eben auch über unbedachte sprachliche Muster. Im Gedächtnis bleiben aufgrund solcher Sprachgebrauchsmuster immer konzeptuelle Spuren zumindest des Verdachts zurück.

Besonders einflussreich wirkt hierbei die massenmediale Kommunikation. Dass persuasive Rhetorik Teil jeder Propaganda in diktatorischen Systemen war und ist, die die Lenkung der öffentlichen Meinung als Ziel hat(te), ist bekannt. Die Betrachtung des Sprachgebrauchs in der NS-Zeit zeigt deutlich, wie eine institutionalisierte Sprachsteuerung Feindbilder schaffen und Weltsichten vermitteln konnte (s. hierzu auch Kap.4.3). Sprachlich vorgegebene Klassifikationen (wie Arier, entartete Kunst, Verjudung, internationales Finanzjudentum) bestimmen maßgeblich Wirklichkeitsauffassungen, kodieren absolute Wertungen. In dem Sinne ist Sprache nicht nur ein wichtiger Träger kulturell-kognitiver Kategorisierungen, sondern auch ein Erzeuger von ihnen. Die Verwendung von Euphemismen (Endlösung, Sonderbehandlung, Euthanasie, judenfrei) dagegen, die nicht semantisch benennen, worauf sie tatsächlich referieren, sondern nebulöse und vage Konzepte aktivieren, dienen der Verharmlosung und De-Realisierung von außersprachlichen Sachverhalten, zumeist von grausamen Gewalttaten.

Aber auch die Massenmedien, die in unserer Demokratie die Informationen verbreiten, haben das Potenzial, Gedanken und Gefühle vieler Menschen ein-

17 Jude war in der NS-Zeit ein Schimpfwort und zwar mit realen Konsequenzen: Die Zuordnung entschied über Bürgerrechte oder Rechtlosigkeit, über Leben und Tod. Wie jüdische Namen als Stigmata benutzt wurden, hat ausführlich Bering (31991 und 2010) gezeigt.

18 Wir haben in den letzten Jahren Umfragen unter Studierenden an deutschen Universitäten durchgeführt, in denen die Denotationen (Grundbedeutungen) und Konnotationen (emotive Zusatzbedeutungen) von Religionsbezeichnungen untersucht wurden. Dabei zeigte sich, dass über 90 Prozent der Befragten Jude (anders als Protestant oder Katholik) nicht als neutrales Appellativum bewerteten, sondern mit Merkmalen wie 'brisant', 'hoch emotional' beschrieben. Diese durch die deutsche Vergangenheit zu erklärenden Attribuierungen stehen also einer Normalisierung bereits konträr gegenüber. Kommen die oben beschriebenen Prozesse hinzu, kann das Wort Jude zusätzliche Merkmale erhalten. 
seitig negativ zu prägen, zumal diese mehrheitlich darauf vertrauen, dass die Mediendarstellungen den Kriterien von Wahrhaftigkeit und Objektivität entsprechen. Wenn die massenmediale Berichterstattung also z. B. über Jahre hinweg emotional einseitig über den Nahostkonflikt berichtet und dabei mittels pejorativer Lexeme und Analogien plakative Opfer- und Täter-Bilder artikuliert, können dadurch Stereotypstrukturen entstehen und alte Ressentiments (re)aktiviert werden (vgl. Kap. 7.3). Gleich, ob Sprache im Alltagsdiskurs oder in der öffentlichen Kommunikation benutzt wird, sie hat stets die Wirkungskraft, das Bewusstsein und die Einstellungen der Rezipienten maßgeblich zu beeinflussen. In dieser Einflussnahme liegt das Machtpotenzial von Sprache.

\subsection{Zur Rekonstruktion von antisemitischen Konzeptualisierungen: Sprachliche Äußerungen als Spuren kultureller, kognitiver und emotionaler Prozesse}

Judenfeindliche Inhalte werden mittels Sprache seit vielen Jahrhunderten ${ }^{19}$ in spezifischer Form repräsentiert und tradiert. Mit sprachlichen Äußerungen werden Stereotype und emotionale Einstellungen ausgedrückt und benannt, geweckt, intensiviert sowie konstituiert. Verbale Ausdrucksrepräsentationen transportieren Ressentiments und vermitteln diese in der Kommunikation als extern wahrnehmbare Bewertungen für Andere. Für das Verständnis von moderner Judenfeindschaft ist es relevant, die Vorstellungen und Bewertungen, die in den sprachlichen Äußerungen zum Ausdruck kommen, zu untersuchen. Bei allen sprachlichen Analysen gehen wir von der Grundannahme (der kognitiven Linguistik) ${ }^{20}$ aus, dass sprachliche Äußerungen Spuren der mentalen Aktivität derjenigen sind, die sie produziert haben. Diese Spuren sind wie Fußabdrücke im Sand: Wir schlussfolgern aufgrund der Beschaffenheit des Abdrucks auf denjenigen, der den Abdruck hinterlassen hat. Entsprechend sind die konkreten Manifestationen Spuren der geistigen Aktivität. Da Sachverhalte der Realität mittels sprachlicher Strukturen stets auf eine spezifische Weise repräsentiert werden, können diese Referenzialisierungen die zugrunde liegenden Konzeptualisie-

19 Die „Ausdrucksformen sind [...] Produkt einer langen historischen Vergangenheit““ (Bering 2004: 378).

20 Die kognitive Linguistik ist eine interdisziplinäre sprach- und kognitionswissenschaftliche Theorie, die sich in den letzten 20 Jahren als der einflussreichste Ansatz der modernen Sprachwissenschaft entwickelt und etabliert hat. Sie basiert auf den Annahmen, dass Sprache ein geistiges Kenntnissystem ist, welches mit anderen Wissenskomponenten interagiert und dass sprachliche Äußerungen Aufschluss über geistige Struktur- und Prozesskomponenten geben (s. u. a. Schwarz ${ }^{3} 2008$ ). 
rungen (im Sinne geistiger Vorstellungen) der Sprachproduzenten vermitteln. ${ }^{21}$ Aufgrund der sprachlichen Manifestationen rekonstruieren wir die zugrunde liegenden geistigen und emotionalen Repräsentationen der Verfasser, die diese Äußerungen erzeugt bzw. motiviert haben. Texte geben somit Aufschluss über die Denkstrukturen, Einstellungen und Gefühle ihrer Verfasser. Judenfeindschaft, als mentale Einstellung und Ressentiment, als kognitive und emotionale Haltung gegenüber Juden, wird über den Sprachgebrauch manifest und damit analysierbar. Somit kann Einblick in den Einstellungs-Antisemitismus gewonnen werden. Bislang spielten jedoch linguistische Analysen (mit wenigen Ausnahmen; vgl. Bering 2004) in der Antisemitismusforschung keine wichtige oder gar herausragende Rolle. Wir werden in diesem Buch zeigen, von welch großer Relevanz sprach- und kognitionswissenschaftliche Detailuntersuchungen sind, da sie präzise und nachvollziehbar formale wie mentale Komponenten der Judenfeindschaft aufdecken und Kriterien für die Klassifikation des verbal tradierten Antisemitismus der Moderne liefern. Da judenfeindliche Denk- und Gefühlsstrukturen heute primär über die Sprache tradiert werden, ist es ein Desiderat, die Charakteristika des antisemitischen Sprachgebrauchs zu analysieren und zu erklären und der internationalen, interdisziplinären Antisemitismusforschung damit einen präzisen Analyse- und Klassifikationsapparat zur Verfügung zu stellen, der wissenschaftliche Kriterien gibt, die festlegen, wann eine Äußerung als antisemitisch einzustufen ist. Angesichts der immer wieder aufs Neue geführten Debatten, der zahlreichen Beleidigungsklagen und sogar Gerichtsprozesse, ist es von nicht unerheblicher Relevanz, wenn die Wissenschaft klar Auskunft darüber geben kann, wann eine Äußerung antisemitisch ist. Zurzeit wird bei nahezu jedem „sprachlichen Delikt“ nach einer Ausrede oder Umdeutung gesucht, gerieren sich die Sprachproduzenten oft als Opfer einer angeblichen „Antisemitismus-Keule“(s. hierzu Kap. 5.2). Eine gesellschaftlich auf breiter Basis bekannte, wissenschaftliche Klassifikation von Verbal-Antisemitismus kann hier Klarheit verschaffen.

In der Regel liegt einer antisemitischen Haltung eine von negativen Emotionen und Stereotypen determinierte Konzeptualisierung von JUDEN ${ }^{22}$ zugrunde.

21 Mit sprachlichen Äußerungen kann man natürlich auch lügen, d. h. bewusst etwas Falsches sagen; man kann seine Vor- und Einstellung auch bewusst verstecken und verschleiern oder sich so indirekt und/oder vage ausdrücken, dass eine eindeutige Festlegung und Rekonstruktion der Intention schwierig, wenn nicht unmöglich ist (zur Lüge als Sprachhandlung s. u. a. Meibauer 2007). Prinzipiell jedoch kann man davon ausgehen, dass die sprachlichen Äußerungen eines Menschen zu einem bestimmten außersprachlichen Sachverhalt viel über seine kognitive Meinung und seine emotionale Einstellung und Verfassung verraten.

22 Konzeptualisierungen stellen kognitive Repräsentationen im Sinne von mentalen Vorstellungsmustern dar, die zu einem bestimmten Welt-Bereich, z. B. einer Person(engruppe), 
Über die Sprache artikuliert sich dann eine solche Konzeptualisierung explizit oder implizit. Ein Satz wie Juden sind Untermenschen macht z. B. die rassistische Ideologie transparent. Ein Satz wie Die Juden wollen die Welt beherrschen gibt Aufschluss darüber, dass der Sprachproduzent offensichtlich das tradierte Stereotyp der JÜDISCHEN WELTVERSCHWÖRUNG in seinem mentalen Glaubenssystem gespeichert hat und von der Wahrheit dieses Glaubensinhalts überzeugt ist. Es gehört zu den Grundprinzipien unserer Kommunikationspraxis, dass wir dem Produzenten stets Kooperativität und damit Rationalität unterstellen, d. h. davon ausgehen, er halte sich an bestimmte Kriterien wie die Maximen der Qualität (Wahrheit) und Relevanz. Wir nehmen also an, dass die Äußerungen genau der Intention des Verfassers entsprechen. Durch Sprache werden im kommunikativen Handeln immer auch rationale und moralische Geltungsansprüche (der Wahrheit, der Richtigkeit und der Aufrichtigkeit) erhoben (vgl. Grice 1975 und Habermas 1981: 525 ff.). Lesen wir Sätze wie „Juden sind dominant im Finanzsektor" (an den ZJD 2002) oder "Juden beherrschen die Presse“ (an die IBD 2007), aktivieren wir nicht nur, ohne dass wir dies in irgendeiner Weise verhindern oder beeinflussen können, die entsprechenden Bedeutungen und Vorstellungen dazu, wir gehen auch automatisch davon aus, dass dies die ehrliche Meinung desjenigen ist, der solche Äußerungen artikuliert. Die Sprache ist also Fenster zum Geist des Sprechers bzw. der „Schlüssel zur Welt des Sprechers“ (Edelman 1976: 168) und zugleich die Straße in den Geist des Hörers (da dieser durch die Äußerungen maßgeblich beeinflusst werden kann, ob er das will oder nicht). Somit spiegelt jede sprachliche Äußerung eine eigene konzeptuelle Text-Welt wider, die der Welt-Ansicht des Produzenten entspricht. Bei der Sprachproduktion wird eine konzeptuelle Struktur fokussiert, die dann den Abruf als angemessen erachteter Wörter steuert. Was als angemessen bewertet wird, hängt von der Intention des Produzenten und auch seiner allgemeinen Einstellung ab. So wird z.B. in einem antisemitischen Text die Bezeichnung Rattenpack für jüdische Mitbürger als adäquat erachtet, da die emotionale Einstellung und Konzeptualisierung hinsichtlich dieser Gemeinschaft von Hass, Abwehr und der Intention der Diskriminierung geprägt sind.

Kognitionslinguistische Textanalysen können einerseits helfen, die Konzeptualisierungsmuster der Textproduzenten transparent zu machen, und andererseits dazu beitragen, das meinungsbildende und vorurteilsverstärkende Potenzial solcher Äußerungen kritisch zu reflektieren. Beim modernen Antisemitismus

gebildet und gespeichert werden (s. Schwarz-Friesel 2007: 81 f., 329 f. und Schwarz ${ }^{3} 2008$ : 114 f.). Den Konventionen der kognitionswissenschaftlichen Darstellungsweise folgend, werden konzeptuelle Entitäten und Strukturen mittels Kapitälchen angezeigt, also das Konzept des Wortes Jude entsprechend als JUDE dargestellt. 
gehen kognitive, emotionale, kulturelle und soziale Aspekte ineinander über. Die zeitlose Komponente der entwertenden Semantik von Judenfeindschaft wird zumeist überlagert von Informationskomponenten, die sich auf aktuelle Geschehnisse (z. B. den Nahostkonflikt, NS-Aufarbeitung, Beleidigungsklagen, Diskussionen) beziehen. Dadurch werden antisemitische Texte von den Zeitgenossen oft nicht als solche erkannt. ${ }^{23}$ Die Rekonstruktion und Erklärung von sprachlicher Evaluierung als Mischung von alten und neuen Informationen ist damit letztlich auch ein Weg, die Interaktion von kulturellem Wissen und emotiven Bewertungsprozessen bei der kommunikativen Tradierung von Judenfeindschaft besser verstehen zu können.

\subsection{Konzeptueller und verbaler Antisemitismus}

Antisemitismus ist ein mentales Glaubens- und Weltdeutungssystem, das seit Jahrhunderten tradiert, aber den aktuellen Gegebenheiten jeweils angepasst und entsprechend modifiziert wird, ohne dass sich die grundlegende konzeptuelle Konstante verändert, der zufolge Juden prinzipiell als DIE ANDEREN fungieren. Dieser konzeptuelle Antisemitismus basiert auf Stereotypen, die geistige Konstruktionen darstellen, d. h. keine empirische Basis haben, sondern reine Projektionen von Nicht-Juden über Juden sind (s. hierzu ausführlich Kap. 4). Wesentlich dabei ist, dass es nicht einzelne Merkmale oder Eigenschaften von Juden sind, die abgelehnt werden. Vielmehr ist es die jüdische Existenz an sich, die als Provokation, als Ärgernis, als Übel in der Welt empfunden wird (s. Kap.9).

$$
\begin{aligned}
& \text { „Verschwindet endlich aus unserer Welt, ihr jüdischer Abschaum!““ } \\
& \text { [ZJD_11.02.2005_Her_003] }
\end{aligned}
$$

23 Dies zeigte sich zuletzt besonders deutlich bei der sehr emotional geführten Diskussion um das Gedicht Was gesagt werden muss von Günter Grass, welches dieser als israel-kritisch und aus Sorge um den Weltfrieden geschrieben deklarierte. Viele Menschen vermochten an diesem Text nichts Antisemitisches zu erkennen, obgleich er nahezu alle tradierten judeophoben Klischees bedient. Da aber das Wort Jude nicht benutzt wurde und die aktuelle Informationskomponente der israelischen Atompolitik in den Vordergrund gerückt wurde, deuteten viele den Text als „nur kritisch“, als „Meinungsfreiheit“ und als „Fakt“. Übersehen wurde dabei, dass es sich um realitätsverzerrende und dämonisierende Äußerungen handelt, die alle typischen Kennzeichen eines modernen antisemitischen Textes im Gewand der IsraelKritik aufweisen (s. hierzu Kap. 7). Moderne Formen des Verbal-Antisemitismus sind oft nur zu erkennen und zu verstehen, wenn die lange Tradition, d. h. die kulturelle und kommunikative Verankerung dieser Feindschaft mit ihren verbalen Strategien, bekannt ist und kontextuell berücksichtigt wird. 


$$
\begin{aligned}
& \text { „Juden - Sie sind das Krebsgeschwür auf unserer Welt!“ } \\
& \text { [IBD_01.08.2006_001_Postkarte] }
\end{aligned}
$$

Judentum ist im mental fixierten, binär strukturierten Weltdeutungssystem von Antisemiten der ultimative Gegenentwurf zur eigenen Existenzform. Entsprechend verkörpern Juden (mit den ihnen angedichteten Eigenarten) das prinzipiell Unnormale und Schlechte. Antisemitismus bedeutet damit, Juden und Judentum über eine absolute und totale Negierung aus der als normal bewerteten Weltordnung auszuschließen. Das grundlegende Kategorisierungsprinzip des menschlichen Geistes ${ }^{24}$ „Omnis determinatio est negatio“ (Spinoza) wird hierbei verabsolutiert: Der Inhalt des Konzeptes JUDE wird als Ganzes ex negativo fixiert und definiert. Über die normalen kontrastierenden Abgrenzungen hinaus, die jeder kognitiven Klassifikation inhärent sind, erhalten Juden im antisemitischen Konzeptualisierungssystem den Status NICHT DAZUGEHÖRIG. JUDE ist somit im kategorialen Sinne eine nicht zu akzeptierende Existenz-Form. Die prinzipielle Entwertung, die mit dieser (unikalen) Kategorisierung einhergeht, spiegelt sich seit Jahrhunderten in zahlreichen Verbalmanifestationen wider, in denen Juden dehumanisiert (Judenschweine, Parasiten), dämonisiert (Monster, Ungeheuer, Teufelsbrut) und delegitimiert (unwertes, schädliches Pack, Endlösung der Judenfrage) werden und damit kognitiv (ipsis litteris gemeint) als „unmenschliche Wesen“ außerhalb der Weltordnung lokalisiert werden. Konzeptueller Antisemitismus ist somit untrennbar gekoppelt an eine de-realisierende Weltsicht (s. zur De-Realisierung Kap. 7.2.1). Das Feindbild JUDE ist ein Konzept jenseits aller Erfahrungswerte, eine Negativ-Konstruktion, die sich aus dem mentalen Glaubenssystem sowie dem tief emotionalen Ressentiment gegenüber Juden ergibt (s. hierzu Kap. 9).

Als Verbal-Antisemitismus gelten alle sprachlichen Äußerungen, mittels derer Juden als Juden entwertet, stigmatisiert, diskriminiert und diffamiert werden, mit denen also judenfeindliche Stereotype kodiert und Ressentiments transportiert werden. Verbal-Antisemitismen sind demnach alle Formulierungen, in denen explizit und implizit Stereotype über Juden ausgedrückt, anti-jüdische Konzeptualisierungen und Gefühle vermittelt und tradierte judeophobe Weltbilder transportiert werden. Konzeptuelle Repräsentationen, die sich aus stereotypen Kategorienkonstrukten zusammensetzen, sind somit immer die Basis für verbal-antisemitische Äußerungen.

Beim intentionalen Verbal-Antisemitismus liegt der Artikulation solcher Äußerungen auf der Konzeptualisierungsebene eine bewusste anti-jüdische Repräsentation zugrunde und der Prozess der Verbalisierung wird durch diese

24 S. hierzu Schwarz/Chur (52007: 38 ff.). 
Konzeptrepräsentation maßgeblich beeinflusst. Zudem liegt die Handlungsabsicht der Äußerung in der intentionalen Diskriminierung und Diffamierung von Juden.

Ob sich der Sprachproduzent verbal-antisemitischer Äußerungen selbst als Antisemit versteht ${ }^{25}$ und/oder ob die Äußerung bewusst und intentional als judenfeindlich artikuliert wird (was letztlich empirisch nur sehr begrenzt rekonstruiert werden kann), spielt für die Klassifikation aber keine Rolle (s. hierzu auch Rensmann/Schoeps 2008: 15). Auch nicht-intentional produzierte VerbalAntisemitismen tradieren judenfeindliche Konzeptualisierungen und bewirken den Erhalt von Stereotypen im kulturellen und kommunikativen Gedächtnis ${ }^{26}$ (zu intentionalem und nicht-intentionalem Verbal-Antisemitismus s. SchwarzFriesel 2007: 347 und 2010a: 30). Bestimmte Floskeln, also Redewendungen wie jüdischer Wucher oder jüdische Hast, kodieren stereotype Vorstellungen zu Juden über Jahrhunderte hinweg, prägen dadurch (zumeist unbewusst und unkontrolliert) kollektive Bewusstseinsprozesse von Gesellschaften; sie sind habitualisiert, werden oft gedankenlos produziert, ohne dass dahinter ein konzeptueller Judenhass steht. Auch die Synonymverwendung der Wörter Jude(n) und Israeli(s) oder eine (über die Semantik eine Unterscheidung in zwei Referenzgruppen ${ }^{27}$ etablierende) Phrase wie Juden und Deutsche muss nicht intentional diskrimi-

25 Es könnte sich auch um einen latenten, d. h. der Person nicht bewussten konzeptuellen Antisemitismus handeln. Im Gedächtnis des Sprachbenutzers sind stereotype anti-jüdische Konzeptualisierungen repräsentiert, die Einfluss auf den Verbalisierungsprozess nehmen; der Sprachproduzent ist sich aber des antisemitischen Potenzials nicht bewusst (z. B. weil das Wissen über anti-jüdische Stereotype und deren Verankerung in abendländischen Denkund Argumentationsstrukturen nicht vorhanden ist oder aber weil er das Bewusstsein, ein Antisemit zu sein, verdrängt, weil es nicht zu seinem Selbstkonzept passt). Davon abzugrenzen sind Inszenierungen, die auf der Strategie der Antisemitismus-Leugnung basieren: Die Sprachproduzenten bezeichnen sich selbst als anti-antisemitisch, drücken aber in ihren Äußerungen klar erkennbar judenfeindliche Stereotype und Gefühle aus (s. hierzu Kap. 11).

26 Das kollektive oder kulturelle Gedächtnis umfasst Wissen, das vielen Mitgliedern einer Gesellschaft bekannt ist und von Generation zu Generation weitergegeben wird. Es beinhaltet nicht nur faktenbasierte Informationen, sondern auch Mythen, Sagen, Glaubensinhalte und Floskeln. Das individuelle Gedächtnis eines jeden Menschen wird maßgeblich von Bestandteilen des kulturellen Gedächtnisses geprägt, und das kollektive Wissen dient vielfach der kulturellen Identifikation. Zum kulturellen Gedächtnis gehören auch Sprachgebrauchsmuster, die im kommunikativen Gedächtnis gespeichert sind. Im 20. und 21. Jahrhundert sind viele Formen von kollektiven und individuellen Gedächtnisinhalten stark emotional und medial geprägt. Vgl. zum kollektiven Gedächtnis u. a. Assmann (1988 und 2006) und zum kommunikativen Gedächtnis Welzer (2002).

27 Äquivalente Phrasen mit additiven Nennungen, die einen kategorialen Unterschied zwischen Gruppen indizieren, wie Deutsche und Katholiken oder Deutsche und Protestanten, werden im Sprachgebrauch nicht benutzt. 
nierend und aggressiv benutzt worden sein. Dennoch tragen solche stereotypverfestigenden Äußerungen dazu bei, dass judeophobe Sprachgebrauchsmuster (mit ihrem Gedankengut) in der Gesellschaft kommunikativ erhalten bleiben. Verbal-Antisemitismen können - anders als die nicht beobachtbaren mentalen Einstellungen oder Absichten, die nur hypothetisch rekonstruiert werden können - mittels objektiver, nachvollziehbarer Kriterien beschrieben und erklärt werden. Als mutmaßlich authentische Spuren der kognitiven und emotionalen Aktivität ihrer Verwender gewähren sie zudem zusätzlich Einblicke in mentale Prozesse und Zustände bzw. lassen es zu, plausible Theorien über diese zu erstellen.

Die Analyse von Äußerungen hinsichtlich ihres verbal-antisemitischen Status und kommunikativen Wertes setzt die Beantwortung von drei Fragen voraus, die drei ${ }^{28}$ verschiedene Aspekte bzw. Analyseebenen involvieren:

1. Ob eine antisemitische Konzeptualisierung zum Ausdruck kommt, also tatsächlich Verbal-Antisemitismus (und nicht lediglich die kommunikative Handlung der Kritik oder der individuellen Beleidigung) vorliegt.

2. Wo der Verbal-Antisemitismus geäußert wird, also im privaten oder öffentlichen Raum (öffentlich/halböffentlich/privat). Wenn es zu einer Verbalisierung von antisemitischen Inhalten kommt, ist von Relevanz, in welcher Diskurssituation und wie öffentlich diese geäußert wird; es geht also um die Frage der Zugänglichkeit und Verbreitung sowie das damit verbundene gesellschaftliche Wirkungspotenzial von antisemitischen Äußerungen.

3. Wie der Verbal-Antisemitismus sprachlich realisiert ist (explizit versus implizit). Das Gegensatzpaar explizit versus implizit benennt die Unterscheidung zwischen direkten und indirekten Sprechakten und damit die Art und Weise, wie in einer Äußerung antisemitisches Gedankengut sprachlich realisiert wird. Explizit wird antisemitisches Gedankengut über die Semantik, d. h. die

28 Ein vierter Analyseschritt betrifft die bereits angesprochene Kategorie „intentional/ nicht-intentional“, die sich aber einer textwissenschaftlichen Untersuchung oft entzieht. Ein eindeutig intentional artikulierter Verbal-Antisemitismus lässt sich z. B. über Äußerungen wie Ich bin Antisemit, und ich hasse alle Juden! bestimmen. Solche expliziten und selbstdefinierenden Aussagen kommen nach 1945 aber fast ausschließlich in rechtsextremen und neo-nazistischen Kreisen vor. Allerdings sind seit einigen Jahren im Internet auch diese direkten Verbal-Antisemitismen nicht nur in extremistischen Foren zu lesen, sondern zunehmend in Chats, Facebook-Seiten, Informationsportalen etc. der sozialen und politischen Mitte zu sehen (Schwarz-Friesel 2012a). Gleichzeitig ist zu konstatieren, dass in rechtsgerichteten Publikationsorganen wie der National-Zeitung und der Jungen Freiheit fast ausschließlich implizite Verbal-Antisemitismen benutzt werden, um ein größeres Publikum persuasiv zu erreichen, das durch explizite, radikale Formen der Judenfeindschaft abgeschreckt werden könnte. 
wortwörtliche Bedeutung ausgedrückt; implizit wird es über die kontextgebundene Äußerungsbedeutung, also die Pragmatik vermittelt und ist über die Schlussfolgerungen zu erschließen (s. hierzu bereits die Ausführungen zu indirekten Sprechakten und zu Implikaturen in Kap. 3.1 und Fußnote 9 in diesem Kap.), die sich zwar aus der Bedeutung ergeben, aber nicht expressis verbis artikuliert werden. Es sind Informationskomponenten sprachlicher Äußerungen, die nicht verbalisiert, vom Rezipienten aber problemlos verstanden werden.

Wenn z. B. in einer E-Mail der ZJD (rhetorisch) gefragt wird, „ob wohl die exzessive Gewalt in Israel, die auch besonders häufig den Mord an Kindern beinhaltet, der langen Tradition Ihres Volkes entspricht“ [ZJD_06.09.2002_Sch_001], werden über Implikaturen die Informationen vermittelt, deutsche Juden seien mitverantwortlich für Gewalt in Nahost und es liege allgemein in der Tradition von Juden, Gewalt anzuwenden. Über den vom Produzenten als wichtig erachteten und daher besonders hervorgehobenen Verweis auf den angeblichen Kindermord wird zudem auf das tradierte judeophobe Stereotyp von JUDEN ALS KINDERMÖRDERN angespielt.

Da sprachliche Äußerungen stets im Kontext und unter Berücksichtigung sowohl des kollektiven Diskurswissens als auch des handlungs- und wirkungsorientierten kommunikativen Sinns zu untersuchen und zu bewerten sind, wäre eine an den bloßen Formen und nur an wörtlichen Bedeutungen orientierte Analyse nicht nur unvollständig, sondern auch unwissenschaftlich.

Die Unterscheidungspaare stehen nicht in einem sich ausschließenden Verhältnis, vielmehr kann eine Äußerung in jeder Kommunikationssituation (öffentlich sowie privat) sowohl explizit als auch implizit Antisemitismus zum Ausdruck bringen.

In der (nicht sprach- und kognitionswissenschaftlichen) Antisemitismusforschung werden diese drei Analyseebenen oft undifferenziert behandelt, terminologisch ausgesprochen heterogen und sehr uneinheitlich mit vagen Termini bezeichnet und oft nicht klar und präzise definiert bzw. voneinander abgegrenzt. Zwischen konzeptuellem und verbalem Antisemitismus wird in der Regel gar nicht unterschieden. Zudem werden beispielsweise die (eher verwirrenden) Begriffe „manifest“ und „latent“ mit wechselnden Lesarten in der Forschungsliteratur verwendet, um (Verbal)Antisemitismus entweder danach zu unterscheiden, ob er in der Öffentlichkeit oder der privaten Kommunikation ${ }^{29}$ artikuliert

29 „Manifest“ und „latent“ werden damit im Sinne der Theorie der Kommunikationslatenz verwendet und beziehen sich auf einen systemtheoretischen Latenzbegriff. Manifest sind demnach alle Formen von Antisemitismus, die öffentlich, also für die Mehrheit bzw. die 
wird oder in welcher Form ${ }^{30}$ er seine Artikulation findet (vgl. etwa Benz 2004: 20). Die Umstände der Kommunikationssituation und die sprachliche Realisierungsform der Äußerung müssen aber als eigenständige Phänomene begrifflich eindeutig bezeichnet und abgegrenzt werden.

Ob eine Äußerung als antisemitisch zu charakterisieren ist, kann unzweideutig mittels text- und diskursanalytischer sowie kognitionslinguistischer Kriterien festgestellt werden (wie wir noch ausführlich im Verlauf dieses Buches zeigen werden). Von alltäglicher Verbal-Aggressivität, die jeden Menschen als Individuum treffen und die sich als Beschimpfung, Verhöhnung, Drohung etc. artikulieren kann, unterscheiden sich Verbal-Antisemitismen wesentlich dadurch, dass die Gruppenzugehörigkeit der Angegriffenen entscheidend für die verbale Gewalt ist: Menschen werden aufgrund ihrer Zugehörigkeit zum Judentum abgelehnt, angegriffen und negativ bewertet, so wie in (7):

„Selbstverständlich hat Möllemann recht! Sharon ist geschichtlich ein Massenmörder! Friedmann ist ein provokanter, intoleranter und arroganter auf gut deutsch ein frecher unverschämter Jude. ReichRanicki kein Literatur-Papst wie von den Medien hoch=gespielt, sondern ein frecher schmieriger polnischer Jude!“ [ZJD_10.06.2002_ano_001]

Nicht individuelle Eigenarten oder Verhaltensweisen sind Grund für die entwertenden Äußerungen, sondern das Jüdisch-Sein der Personen. Das konzeptuelle Merkmal JÜDIsCH ist stets ausschlaggebend und die mentale Basis für die verbal vollzogene Klassifikation und Bewertung. Damit sind Verbal-Antisemitismen spezifische Formen der sprachlichen Diskriminierung (s. 3.2 in diesem Kap.). Die mentale Fixierung, die über das Konzept JUDE zustande kommt, kann personenbezogen wie in (7), aber auch de-personalisiert vollzogen werden. Dann werden als typisch jüdisch klassifizierte Handlungsmuster angeführt wie z. B. in (8), wo

\footnotetext{
Gesellschaft beobachtbar sind, wohingegen latenter Antisemitismus nicht Teil der öffentlichen Kommunikation ist und nur in kleineren und privaten Diskursen auftritt. Wesentlich klarer wird dies mit dem Begriffspaar „öffentlich“versus „privat“ bezeichnet.

30 So bezeichnen u. a. auch Pollak/Eger (2002) und Stern (2002) impliziten VerbalAntisemitismus, also die Vermittlung judenfeindlichen Gedankengutes über indirekte Sprechakte, als „latente Form des Antisemitismus“. S. auch Rensmann/Schoeps (2008: 16) und die Beiträge in Pelinka/Wodak (2002). Bergmann/Erb (1986) bezeichnen diese Phänomene dagegen als „Umwegkommunikation“ (vgl. auch Rensmann 2004: 78 f.). Adorno ([1962] 1971: 109) benutzte seinerzeit den Begriff des „Krypto-Antisemitismus“. Die sprachund kommunikationswissenschaftlichen Begriffe „explizit“ und „implizit“ sind für die verschiedenen Realisierungsvarianten aber am besten geeignet. Sie haben sich in Jahrzehnten theoretischer und empirischer Sprach- und Diskursanalysen bewährt.
} 
explizit durch das Wort „jüdisch“ und in (9), wo implizit über den Verweis auf die „zwei Tausend Jahre“ eine Verbindung zwischen israelischen Militäraktionen und jüdischer Mentalität gezogen wird:

„Der Herr Botschafter hat dieser Tage bestritten, daß die Luftwaffe des Judenstaates auf ein deutsches Aufklärungsschiff geschossen hat. [...] Der Herr Botschafter hat also nur getan, was ihm die jüdische Tradition seit jeher erlaubt.“[IBD_31.10.2006_Koe_001]

„Seit zwei Tausend Jahren betreiben Sie Landraub und Mord!“

[IBD_11.09.2007_Mar_001]

Gerade bei den modernen Formen der über eine referenzielle Verschiebung (vom Referenzobjekt Jude zum Referenzobjekt Israel) realisierten Verbal-Antisemitismen werden primär diese de-personalisierten Stereotypzuweisungen vollzogen (s. hierzu ausführlich Kap. 7).

Verbal-Antisemitismen gibt es in zahlreichen Ausprägungsvarianten als Beschimpfungen, Holocaustleugnungen, Gewaltandrohungen, NS-Vergleiche, Unterstellungen und Anspielungen mittels rhetorischer Fragen, Zitat-Anführungen usw. Dabei ist zu zeigen, wie groß die Palette der Möglichkeiten ist, antijüdische Stereotype und Gefühle zu kodieren; auf vielerlei Weise und in den verschiedensten Formen können judeophobe Inhalte verbal vermittelt werden. Die als typisch (verbal-)antisemitisch geltenden Beschimpfungen wie Wucherjuden, die expliziten Holocaustleugnungen wie in Auschwitzlüge und die Stereotypzuschreibungen mittels generischer Sätze wie in Alle Juden sind geldgierig stellen nur einen sehr kleinen Teil der mannigfaltigen Typen von Verbal-Antisemitismen dar. Bei allen lexikalischen, grammatischen und stilistischen Differenzen, bei allen Nuancen und Variationen in den Realisierungsformen lassen sich (neben der bereits genannten Semantik und Pragmatik von Dehumanisierung und Delegitimierung, die sich aus der konzeptuellen De-Realisierung ergibt) einige grundlegende kategoriale Eigenschaften von Verbal-Antisemitismus benennen: kollektive Abgrenzung, Fixierung und Entwertung. ${ }^{31}$ Die Abgrenzung von Juden kann sprachlich auf vielerlei Weisen indiziert werden (ausführlich hierzu Kap. 5):

31 S. hierzu auch Graumann/Wintermantel (2007), die als typische Kennzeichen für verbale Diskriminierung die Prozesse des Trennens, Akzentuierens/Fixierens, Abwertens/De-

Evaluierens anführen. Vgl. auch Haubl/Caysa (2007: $22 \mathrm{f}$.), die als wesentliche Merkmale für die Emotion Hass die Entwertung des Hassobjekts und die Abgrenzung des Gehassten nennen. 
„Können Juden Deutsche nicht endlich in Ruhe lassen?“

[ZJD_29.07.2006_Bur_001]

Grundlage ist dabei stets die Konzeptualisierung, Juden seien prinzipiell anders und keineswegs der Eigengruppe zugehörig. Die unterstellte Anders- oder Fremdartigkeit wird mittels negativer Stereotypzuordnung fixiert und damit legitimiert bzw. (pseudo)rationalisiert.

$$
\text { „Ihr Juden ward und seit Diebe, Mörder, Betrüger, Landräuber!“ }
$$$$
\text { [IBD_05.08.2008_Dro_001] }
$$

Eine Variante dieses verbalen Antisemitismus liegt vor, wenn Stereotypzuordnungen ausgedrückt werden, wobei vom Individuum bzw. von Individuen auf die gesamte Gruppe aller Juden geschlossen wird, z. B. in Äußerungen wie (12) und (13):

$$
\begin{aligned}
& \text { „Das gilt besonders für Ihren überaus arroganten und anmaßenden Vice, } \\
& \text { Friedmann. Seine gesamte Art und Weise, wie er auftritt, und was er von } \\
& \text { sich gibt, löst in mir gelinde gesagt, ein Unbehagen gegenüber dem } \\
& \text { Judentum aus, was ich bisher in keinster Weise kannte.“ }
\end{aligned}
$$

[ZJD_18.06.2002_Neu_001]

(13) „Ihr Israelis seid ein menschenverachtender Haufen. Werft in den letzten Kriegstagen Streubomben über bewohntem Gebiet ab, und werft den Menschen, die sowas kritisieren, Antisemitismus vor. Das ist typisch für Euch Juden!“‘[ZJD_01.09.2006_Wei_001]

Juden werden aufgrund ihrer Gruppenzugehörigkeit kollektiv auf bestimmte Merkmale, Eigenschaften und Verhaltensweisen festgelegt, also über Stereotype in einer konzeptuell geschlossenen Kategorie fixiert. Diese Kategorie wird als prinzipiell negativ bewertet. Mit jeder Kollektivattribuierung geht also beim Antisemitismus eine Entwertung einher. Diese Entwertung ist signifikant oft an die Semantik der Dehumanisierung geknüpft:

(14) „Kein menschlich denkendes Wesen kann auf eurer Seite sein. [...] Man wird Euch [...] bekämpfen wo und wie es nur geht.“

[IBD_20.02.2009_Gut_001] 
„Seid ihr ueberhaupt Menschen? Ihr zeigt nicht einmal das Sozialverhalten von Tieren, Ratten oder Mikroben!“

[ZJD_Gaza2009_300/816_Mon_001]

Verbaler Antisemitismus liegt vor, wenn eine völkisch-rassistische Definition die Basis bei der Zuordnung „Jüdisch-Sein“ ist, in dem Sinne, dass Jude immer Jude bleibt und dass Jüdisch-Sein untrennbar an bestimmte, unveränderliche Merkmale oder Eigenschaften gekoppelt ist.

$$
\begin{aligned}
& \text { „Ihr Juden ändert euch nie. Mieses Volk!!!“ [IBD_07.11.2006_ano_001] } \\
& \text { „Juden sind halt so seit über } 2 \text { Tausend Jahren.“ } \\
& \text { [ZJD_12.03.2007_Kli_001] }
\end{aligned}
$$

Verbaler Antisemitismus liegt auch vor, wenn in den Äußerungen die Verantwortung der Deutschen für den Holocaust geleugnet oder relativiert wird, wenn historische Fakten, die die Judenvernichtung betreffen, verzerrt oder falsch dargestellt werden, wenn eine Täter-Opfer-Umkehr ausgedrückt oder angedeutet wird, wenn für das Ende der Erinnerungs- und Verantwortungskultur argumentiert wird und NS-Vergleiche benutzt werden:

(18) „Aber die Israelis ermorden kleine Kinder, eine Schande soetwas. Schlimmer als der Holocaust, falls es diesen überhaupt gegeben hat. Die schlimmsten Verbrecher der Welt sind die Juden. Das schlimme daran ist, dass die Juden sich an sowas noch erfreuen. Einfach eine Schande was die Juden sich auf der Welt leisten. Erst heulen wegen Hitler und dann jetzt dasgleiche machen, lächerlicher gehts kaum. In diesem Sinne PRO HAMAS und gegen die verbrecherische JUDENBANDE.“ [ZJD_Gaza2009_153/816_Rie_001]

(19) „Hallo ihr Juden, [...] Zu den vielen Lügen, wie auch die, der 6 Millionen 'Holocaust'-Opfer. Euer eigenes Yad Vashem hat bis heute nur 3 Mio. Namen zusammengetragen. Auch eine dieser lügen, für 6 Millionen kassieren - typisch Juden. Passt genau in das Clichee dasss über euch besteht.“[IBD_28.10.2006_ano_001]

(20) „Den jüdischen Zentralrat hat keiner gewählt! Wieso bezahlen wir eigentlich immer noch Wiedergutmachung was vor über 50 Jahre passiert ist??? Die Schuld ist beglichen!!!!!“ [ZJD_26.08.2007_Kar_001] 
Verbaler Antisemitismus liegt vor, wenn die Begriffe „Israel(is)“, „Zionismus“/ „Zionisten“ und „Juden(tum)“ gleichgesetzt oder vermischt werden, um eine kritische oder feindselige Haltung allen Juden gegenüber auszudrücken.

(21) „Hut ab vor dem jüdischen Volk. Sie haben gut von Hitler und seinem NS-Regime gelernt. Denn das was die Zionisten heute mit den Palistinensern machen ist auch nicht besser wie das, was sie den Deutschen immer wieder vorwerfen, um von Ihren eigenen Greueltaten abzulenken.“ [ZJD_09.03.2008_Stra_001]

(22) „Wenn unschuldige, kleine Kinder, Mütter und Väter durch Angriffe von Israel ums Leben kommen, das ist viel schlimmer, als wenn hin und wieder anti-jüdische Äusserungen getätigt werden! [...] ich schäme mich für alle Juden.“[ZJD_05.01.2009_Kil_001]

Es ist auch antisemitisch, die israelische Politik allen Juden anzulasten und kollektiv Schuld zuzuweisen:

(23) „Sie persönlich Herr Spiegel, unterstützen, befürworten und dulden in Israel ein Massaker. Sie machen sich schuldig, Herr Spiegel.“

[ZJD_08.04.2005_Hah_001]

Hier handelt es sich um eine unzulässige Generalisierung der Art 'Juden sind verantwortlich für Aktivitäten des israelischen Staates'. Man würde ja z. B. nie allen Katholiken die Politik des Vatikans anlasten. Diese kollektiven Schuldzuweisungen basieren zudem auf dem alten antisemitischen Stereotyp JUDEN SIND KEINE DEUTSCHEN (in der aktuellen Variante JUDEN SIND ISRAELIS).

Verbaler Antisemitismus liegt vor, wenn in der Referenz auf Israel negative judeophobe Stereotype übertragen und Argumente benutzt werden, um generelle Negativzuschreibungen und jüdisch-israelische Gleichsetzungen sowie Generalisierungen zu erzielen.

(24) „Christusmörder, Diebe, Betrüger, Judenpack: ‘auserwähltes Volk’““ [IBD_31.07.2006_Luh_001]

(25) „Befreit den nahen Osten von der jüdischen Pest!“ [IBD_12.07.2006_ano_003] 
„soll iran die atombombe bekommen und israel von der karte bomben. das ist was dieses scheisspack von kindermördern verdient. fahrt allesamt zur hölle ihr jüdischen arschlöcher.“ [ZJD_31.01.2006_ano_001]

In den nachfolgenden Kapiteln werden wir ausführlich auf die verschiedenen Verbalmanifestationen von Judenfeindschaft eingehen, ihre Charakteristika anhand zahlreicher Beispiele erörtern und in ihrem historischen Diskurskontext erklären sowie die konzeptuellen und emotionalen Repräsentationen transparent machen, die diesen sprachlichen Mustern zugrunde liegen.

\section{Fazit}

Sprachliche Äußerungen bilden nicht nur außersprachliche Realität ab, sie erzeugen zum Teil auch über die Semantik eigene Realitäten. Judeophobe Sprachgebrauchsmuster konstituieren und tradieren im kollektiven kommunikativen Gedächtnis mentale Modelle, in denen Juden konzeptuell als DIE ANDEREN repräsentiert werden. Verbale Judenfeindschaft zeichnet sich durch eine destruktive Semantik aus, die auf den Prozessen der Abgrenzung, der Stereotypfestlegung und der Entwertung basiert. Wesentlich dabei ist, dass es nicht nur einzelne Merkmale oder Eigenschaften von Juden sind, die abgelehnt werden. Vielmehr ist es die jüdische Existenz an sich, die als Provokation, als Ärgernis, als Übel in der Welt empfunden wird. Das antisemitische Ressentiment ist auf kein bestimmtes, konkretes Referenzobjekt in der realen Welt ausgerichtet, sondern bezieht sich auf das im Kopf der Sprachproduzenten gespeicherte Konzept JUDE, das keine empirische Fundierung hat. 